\title{
Análise Comparativa do Tempo de Vida de Baterias em Dispositivos Móveis a partir da Utilização de Modelos Analíticos ${ }^{1}$
}

K.K. SCHNEIDER 2 , P.S. SAUSEN ${ }^{3}$, A. SAUSEN ${ }^{4}$, UNIJUÍ - Universidade Regional do Noroeste do Estado do Rio Grande do Sul, Mestrado em Modelagem Matemática, Ijuí, RS, Brasil.

Resumo. Neste artigo é apresentada uma análise comparativa entre dois modelos analíticos de descarga de baterias: o modelo Linear e o modelo de RakhmatovVrudhula. Esta análise tem como objetivo verificar qual modelo é mais adequado para a estimação do tempo de vida de uma bateria alcalina na alimentação de nós sensores utilizados em Redes de Sensores Sem Fio (RSSF). Desta maneira, foram realizadas simulações considerando os parâmetros da bateria alcalina e as especificações de um nó sensor da família Mica Motes. A partir da análise dos resultados das simulações verificou-se que o modelo de Rakhmatov-Vrudhula apresentou um decrescimento menor na capacidade da bateria quando comparado com o modelo Linear, pois consegue capturar os efeitos não-lineares que ocorrem durante as operações de descarga.

Palavras-chave. Modelos Matemáticos, tempo de vida, efeito de recuperação.

\section{Introdução}

Nas últimas décadas, o número médio de dispositivos móveis (e.g., telefones celulares e notebooks) por cidadão tem aumentado significativamente devido, principalmente, à proliferação no acesso à tecnologia sem fio. Tais dispositivos são alimentados por algum tipo de bateria, geralmente recarregável, cuja função é o fornecimento de energia ao sistema. Destaca-se que a utilização destes dispositivos está condicionada ao tempo de vida das baterias que os alimentam. Este tempo de vida é por definição o tempo que a bateria demora para atingir um determinado nível de capacidade de carga (i.e., nível de cutoff), ao alcançar este nível as reações eletroquímicas, responsáveis pelo fornecimento de energia, cessam e consequentemente a bateria deixa de fornecer energia ao sistema, sendo considerada descarregada. Neste contexto, é de vital importância possuir algum método capaz de predizer o tempo de vida da bateria e por conseguinte o comportamento dinâmico do sistema como um todo.

\footnotetext{
${ }^{1}$ Os autores deste trabalho agradecem a CAPES e a FAPERGS (Edital 03/2009) pelo aporte financeiro recebido.

${ }^{2}$ keilaschneider@hotmail.com; Mestranda do curso de Modelagem Matemática da UNIJUÍ.

${ }^{3}$ sausen@unijui.edu.br; Professor Orientador

4airam@unijui.edu.br; Professora Co-orientadora
} 
Uma das formas de realizar a predição do tempo de vida de baterias é utilizar um modelo matemático que representa adequadamente o processo, ou seja, que descreva a descarga de energia do sistema. Diferentes modelos de baterias têm sido desenvolvidos ao longo dos anos. Destacam-se os modelos eletroquímicos, os modelos de circuitos elétricos, os modelos estocásticos, e os modelos analíticos [3, $4,5]$. Estes últimos descrevem a bateria em um nível mais elevado de abstração, de modo que as suas principais propriedades são modeladas utilizando um conjunto reduzido de equações, tornando a implementação mais simples, quando comparado com os modelos citados anteriormente.

Os modelos analíticos são computacionalmente eficientes e flexíveis, requerendo avaliação de simples expressões analíticas, podendo ser facilmente configurados para diferentes tipos de baterias. Eles podem incluir a corrente como uma função de carga constante ou de carga variável. O modelo analítico mais simples é o modelo Linear [3, 4, 11], que considera apenas correntes de descargas constantes no tempo, modelando a bateria como um recipiente de energia, onde a corrente é decrementada de forma linear. Há também a Lei de Peukert [4, 5, 6, 9], que considera o tempo de vida da bateria sob cargas variáveis através da média das correntes, resultando em tempos de vida muito parecidos com os tempos de vida do modelo linear.

Por outro lado, a utilização de modelos lineares não permite que sejam realizadas aproximações satisfatórias, especialmente quando são considerados os aspectos físicos das operações de descarga da bateria. Estudos recentes $[3,4,5]$ têm revelado que as taxas de descarga são não-lineares no tempo e, além disso, dependem da capacidade residual da bateria e da intensidade da corrente de descarga (efeito da taxa de capacidade), ou seja, para diferentes perfis de descarga têm-se diferentes tempos de vida, assim, a capacidade efetiva da bateria não é a mesma para diferentes perfis de descarga. Entende-se por perfil de descarga o conjunto de operações que um certo dispositivo pode realizar em um determinado intervalo de tempo. Por exemplo, um sensor pode receber e transmitir informações, sendo que para receber tem-se uma taxa de consumo diferente da taxa de transmissão. Além disso, em períodos ociosos (i.e., quando a corrente de descarga é reduzida significativamente ou mesmo é nula), ocorre um sutil, mas importante efeito de recuperação, que pode recuperar a capacidade da bateria [3].

Neste contexto, ressalta-se a importância da utilização de um modelo matemático acurado que considera, entre outras características, as características nãolineares e seus efeitos na capacidade da bateria, especialmente quando o objetivo final é predizer o tempo de vida da mesma. Neste trabalho será realizada uma comparação entre o modelo analítico de difusão de Rakhmatov-Vrudhula [4, 10] e o modelo Linear [11], na estimação do tempo de vida de uma bateria. O modelo de Rakhmatov-Vrudhula foi escolhido em virtude do mesmo conseguir capturar o efeito de taxa de capacidade e o efeito de recuperação, bem como ser de fácil implementação quando comparado aos demais modelos citados [11].

O restante deste trabalho está organizado como segue. Na Seção 2. são apresentadas algumas características e conceitos básicos sobre baterias. Na Seção 3. é descrita a modelagem matemática do processo. Na Seção 4. são apresentados os resultados das simulações entre o modelo Linear e o modelo de Rakhmatov-Vrudhula e sua análise. E por fim, na Seção 5. são apresentadas as conclusões. 


\section{Baterias}

Uma bateria é constituída por uma ou mais células eletroquímicas, ligadas em série ou em paralelo, ou ainda através de uma combinação de ambas. Nestas células a energia química armazenada é convertida em energia elétrica através de reações eletroquímicas. Uma bateria pode ser representada através de uma célula eletroquímica (Figura 1), onde cada célula é formada por dois eletrodos ${ }^{5}$ : um ânodo que possui polaridade negativa e um cátodo que possui polaridade positiva, separados por um eletrólito ${ }^{6}$. Durante a fase de descarga (i.e., quando a bateria fornece corrente elétrica a um circuito externo) o ânodo libera elétrons para o circuito, enquanto o cátodo recebe elétrons do circuito. Estes elétrons têm origem a partir de reações eletroquímicas e são chamados de espécies eletroativas [4, 11].

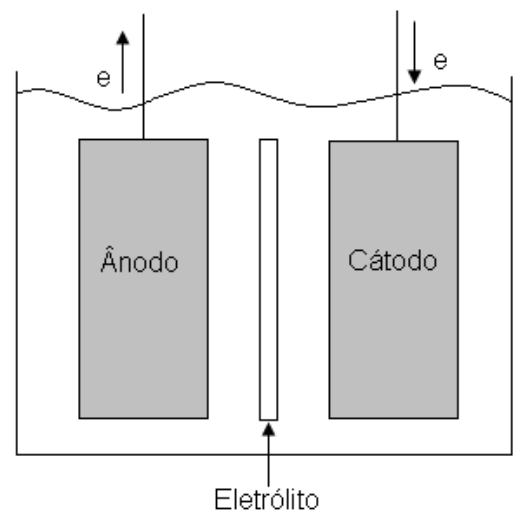

Figura 1: Esquema de uma célula eletroquímica [4].

\subsection{Propriedades das baterias}

Uma bateria possui duas importantes propriedades, que são: (i) a voltagem, medida em volts (V); e (ii) a capacidade, geralmente medida em ampere-hora (Ah). Assim, teoricamente, uma bateria de 100 Ah é projetada para fornecer 5 A por 20 horas, ou então, 100 A durante 1 hora. Porém, esta representação é teórica, na prática, geralmente, a corrente de descarga não é constante no tempo, ou seja, as operações de descarga têm características não-lineares e consequentemente ocorrem efeitos não-lineares que influenciam diretamente no tempo de vida da bateria. Estes efeitos serão descritos na próxima seção.

\subsection{Características não-lineares}

A modelagem do comportamento de baterias pode se tornar complexa se considerados os efeitos não-lineares que ocorrem durante o período de descarga. No caso ideal, a tensão permanece constante durante todo o período de descarga, tornandose zero quando a bateria está descarregada. O ideal seria que a capacidade fosse

\footnotetext{
${ }^{5}$ Condutor metálico por onde uma corrente elétrica entra ou sai do sistema.

${ }^{6}$ Condutor de eletricidade, sólido ou líquido, onde o transporte de carga se realiza por meio de íons.
} 
constante para qualquer corrente de descarga, e que toda a energia armazenada na bateria fosse utilizada. Contudo, em um procedimento de descarga de bateria real, a tensão diminui lentamente durante a descarga e a capacidade efetiva é reduzida para altas correntes. Ressalta-se que, dependendo do tipo de bateria, estes efeitos têm maiores ou menores consequências na sua capacidade [3, 4]. Alguns dos efeitos não-lineares são apresentados nas próximas seções.

\section{(A) Efeito de Recuperação}

Define-se por efeito de recuperação de uma bateria a reorganização dos elétrons no eletrólito durante um período de relaxação, ou seja, intervalo de tempo em que a corrente de descarga é reduzida significativamente. Neste intervalo os elétrons se reorganizam de maneira uniforme, de modo que o sistema recupere o equilíbrio e o gradiente de concentração seja nulo na superfície do eletrólito. Assim, a capacidade efetiva da bateria é aumentada, pois uma maior quantidade de carga torna-se disponível antes do sistema alcançar o nível de cutoff (i.e., quando a bateria está descarregada). Na Figura 2 são mostradas, de forma simplificada, as operações de uma bateria, onde o efeito de recuperação pode ser observado.

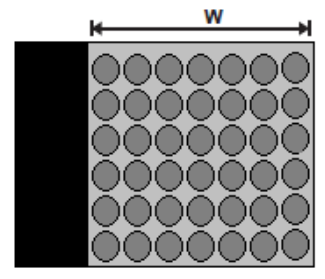

(A) Totalmente Carregada

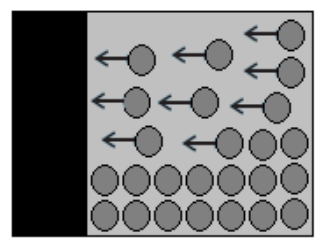

(C) Em Recuparação

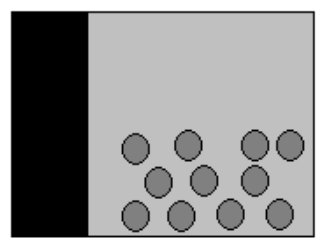

(E) Bateria Descarregada

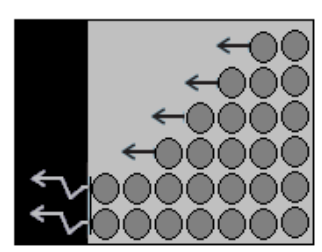

(B) Descarregando

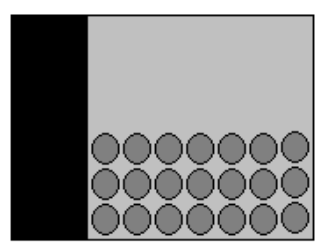

(D) Após Recuperação

Legenda

Espécies Eletroativas

$\leftarrow$ Difusão

«. Consumo

$\square$ Eletrólito

Eletrodo

Figura 2: Diferentes estados de operação da bateria [11].

Na Figura 2(A), percebe-se que a bateria está totalmente carregada e a concentração de espécies eletroativas é constante por todo comprimento $w$ do eletrólito. Quando um descarregamento é iniciado (Figura 2(B)), ocorrem reações eletroquímicas que resultam na redução de espécies eletroativas próximas ao eletrodo, gerando um gradiente de concentração no eletrólito. No momento em que a corrente de 
descarga é reduzida significativamente ou é nula (i.e., dispositivo desligado), a bateria encontra-se em um período de relaxação (Figura $2(\mathrm{C})$ ), possibilitando que os elétrons se reorganizem de maneira uniforme, reequilibrando o sistema. Assim, é gerado um gradiente de concentração nulo no eletrólito, tornando disponível uma maior quantidade de carga na superfície do eletrodo, que agora pode ser utilizada pelo sistema, aumentando assim a capacidade efetiva da bateria, como pode ser observado na Figura 2(D). À medida que a concentração diminui, a tensão da bateria é reduzida. Quando a tensão da bateria atinge um limite inferior ao valor de corte (i.e., cutoff) as reações eletroquímicas não podem mais ocorrer e a bateria para de fornecer carga ao sistema. Neste momento a bateria pode ser considerada descarregada, apesar de ainda haver espécies eletroativas no eletrólito, conforme mostrado na Figura 2(E).

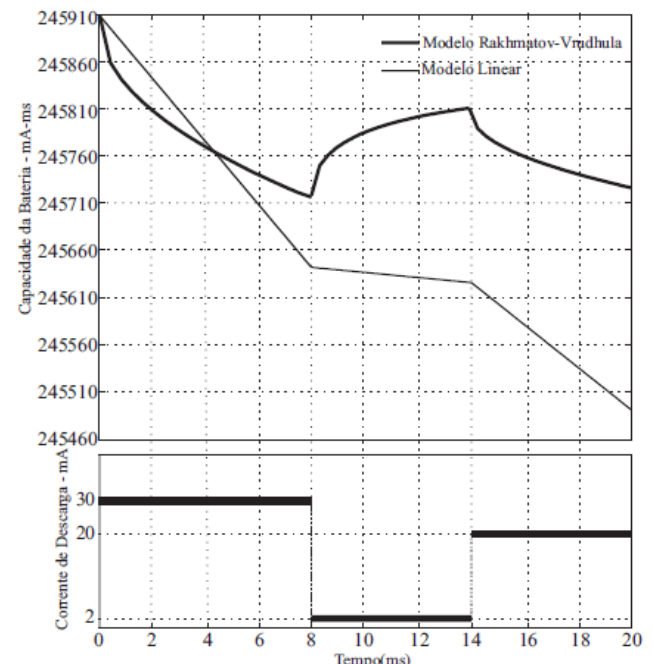

Figura 3: Capacidade da bateria em função do perfil de descarte [11].

Para melhor explicar o efeito de recuperação, na Figura 3 é apresentada a capacidade de uma bateria capturada pelo modelo de Rakhmatov-Vrudhula e pelo modelo linear, considerando um perfil de descarga representado pelo vetor de corrente $I=\left[\begin{array}{lll}30 & 2 & 20\end{array}\right]^{T}$ em $m A$ e pelo vetor tempo $t=\left[\begin{array}{ccc}8 & 6 & 6\end{array}\right]^{T}$ em $m s$. Desta forma, a descarga é de 30,2 e $20 \mathrm{~mA}$ para os intervalos de tempo de 8, 6 e $6 \mathrm{~ms}$, respectivamente. Pode-se verificar o efeito de recuperação (entre o intervalo de tempo de 8 e $14 \mathrm{~ms}$ ), capturado pelo modelo de Rakhmatov-Vrudhula, quando ocorre uma redução na corrente de $30 \mathrm{~mA}$ para $2 \mathrm{~mA}$. Considerando o modelo linear, para o mesmo intervalo de tempo, verifica-se apenas o consumo de energia, já que este não captura o efeito de recuperação. Percebe-se que para ocorrer o efeito de recuperação, não é necessário que o dispositivo seja desligado (i.e., carga drenada na bateria é zero), basta apenas que a corrente solicitada da bateria seja reduzida significativamente por um determinado intervalo de tempo. No caso do exemplo, apresentado na Figura 3, a redução na corrente ocorreu de $30 \mathrm{~mA}$ para 2 $m A$ durante 6 ms. 
(B) Nivel Cutoff

O nível de cutoff [11], apesar de não ser um efeito não-linear, é um importante parâmetro para compreensão do efeito de recuperação. Ele pode ser definido como o valor limite de carga (capacidade) em que a bateria consegue fornecer uma tensão constante. Quando este valor é atingido, a bateria não é mais capaz de fornecer energia ao sistema devido à impossibilidade de ocorrer as reações eletroquímicas. Neste momento a bateria não está completamente descarregada, mas indisponível, como pode ser visto na Figura 2(E).

(C) Efeito da Taxa de Capacidade

O efeito da taxa de capacidade $[4,11]$ depende da capacidade atual da bateria e da intensidade da corrente de descarga, ou seja, com uma alta corrente de descarga a capacidade efetiva da bateria é baixa, pois não há tempo suficiente para a reorganização dos elétrons no eletrólito (i.e., efeito de recuperação) reduzindo, desta forma, a capacidade da bateria. Já com correntes alternadas, a capacidade efetiva da bateria é aumentada, pois na troca de uma corrente alta para uma corrente baixa, ou até mesmo para um período sem corrente, ocorre a reorganização dos elétrons no eletrólito (i.e., efeito de recuperação), aumentando, assim, a capacidade efetiva da bateria. Ressalta-se que esta capacidade nunca será maior, nem igual, à capacidade inicial.

\subsection{Capacidade da bateria}

A capacidade de uma bateria é especificada pela quantidade de materiais ativos nela presente. Esta capacidade pode ser expressa de três maneiras diferentes [5, 11]: (i) Capacidade Teórica, é o limite máximo de energia que pode ser extraída na prática sendo baseada na quantidade de energia armazenada; (ii) Capacidade Padrão é a energia que pode ser extraída sob condições especificadas pelo fabricante; (iii) Capacidade Real é aquela que pode exceder a capacidade padrão, mas não pode exceder a capacidade teórica de uma bateria.

O desempenho de uma bateria em relação ao perfil de corrente de descarga depende de dois efeitos: (i) efeito de taxa de capacidade, que depende da capacidade atual da bateria, e da intensidade da sua corrente de descarga; (ii) efeito de recuperação, que depende da recuperação da capacidade durante períodos ociosos, ou seja, períodos em que a corrente solicitada é reduzida significativamente.

\section{Modelagem Matemática}

Nesta seção é apresentado o modelo matemático analítico de Rakhmatov-Vrudhula que, entre outras características, considera os efeitos não-lineares da bateria. $\mathrm{O}$ processo de difusão unidimensional do modelo é descrito pelas leis de Fick dadas pelo sistema de Equações Diferenciais Parciais (EDPs) apresentado a seguir

$$
\begin{aligned}
& -J(x, t)=D \frac{\partial C(x, t)}{\partial x}, \\
& \frac{\partial C(x, t)}{\partial t}=D \frac{\partial^{2} C(x, t)}{\partial^{2} x}
\end{aligned}
$$


onde $J(x, t)$ é o fluxo de espécies eletroativas em função do tempo $t$ e em função de uma distância $x$ do eletrodo, $D$ é a constante de difusão, e $C(x, t)$ é a função concentração de espécies eletroativas no tempo $t \in[0, L]$ e na distância $x \in[0, w]$. Para uma bateria completamente carregada (i.e., $t=0$ ) a concentração de espécies eletroativas é constante através do comprimento do eletrólito, proporcionando a seguinte condição inicial

$$
C(x, 0)=C^{*}
$$

A bateria é considerada descarregada quando $C(0, t)$ é inferior ao nível de cutoff. De acordo com a Lei de Faraday, o fluxo na superfície do eletrodo $(x=0)$ é proporcional à corrente $i(t)$ (i.e., carga externa aplicada). Por outro lado, por simplificação de modelagem, o fluxo na outra extremidade da região de difusão $(x=w)$ é considerado zero. Estas proposições fornecem as seguintes condições de fronteira

$$
\begin{gathered}
\frac{i(t)}{v F A}=\left.D \frac{\partial C(x, t)}{\partial x}\right|_{x=0}, \\
0=\left.D \frac{\partial C(x, t)}{\partial x}\right|_{x=w},
\end{gathered}
$$

onde $A$ é a área da superfície do eletrodo, $F$ é a constante de Faraday, e $v$ é o número de elétrons envolvidos na reação eletroquímica na superfície do eletrodo.

Através da aplicação da transformada de Laplace e da transformada de Laplace inversa na EDP apresentada na equação (3.2) obtém-se a solução $C(x, t)$. Porém, é desejado somente a concentração de espécies eletroativas na superfície do eletrodo em $x=0$, sendo encontrado que

$$
C(0, t)=C^{*}-\frac{1}{v F A \sqrt{\pi D}} \int_{0}^{t} \frac{i(\tau)}{\sqrt{t-\tau}} \sum_{m=-\infty}^{\infty} e^{-\frac{w^{2} m^{2}}{D(t-\tau)}}
$$

Dividindo a equação (3.6) pela condição inicial $C^{*}$ e considerando $\rho(t)=1-$ $C(0, t) / C^{*}$ a fração de decaimento da concentração de espécies eletroativas na fronteira $x=0$, a equação (3.6) torna-se

$$
\rho(t)=\frac{1}{v F A \sqrt{\pi D} C^{*}}\left[\int_{0}^{t} \frac{i(\tau)}{\sqrt{t-\tau}} d \tau+2 \sum_{m=1}^{\infty} \int_{0}^{t} \frac{i(\tau)}{\sqrt{t-\tau}} e^{-\frac{w^{2} m^{2}}{D(t-\tau)}} d \tau\right] .
$$

Sendo $\beta=\frac{w}{\sqrt{D}}$ o parâmetro que está relacionado ao comportamento não-linear, e $\alpha=v F A \sqrt{\pi D} C^{*} \rho(L)$ o parâmetro que está relacionado à capacidade da bateria, obtém-se a seguinte expressão geral

$$
\alpha=\int_{0}^{L} \frac{i(\tau)}{\sqrt{L-\tau}} d \tau+2 \sum_{m=1}^{\infty} \int_{0}^{L} \frac{i(\tau)}{\sqrt{L-\tau}} e^{-\frac{\beta^{2} m^{2}}{(L-\tau)}} d \tau
$$

que relaciona o tempo de vida $L$ para um perfil de carga $i(t)$, onde os parâmetros $\beta$ e $\alpha$ necessitam ser estimados. Neste trabalho será utilizado os parâmetros de uma bateria alcalina, estimados a partir da técnica de mínimos quadrados [11]. 
Em seguida, considera-se uma taxa de descarga variante no tempo, aproximada por uma carga constante por partes, também chamada de função de $n$ degraus [9], dada por

$$
i(t)=\sum_{k=1}^{n} I_{k-1}\left[U\left(t-t_{k-1}\right)-U\left(t-t_{k}\right)\right]
$$

onde $I_{k}$ é a carga constante; $U(t)$ é uma função degrau, com $U(t)=1$ se $t>0$, enquanto $U(t)=0$ se $t<0$. Substituindo a equação (3.9) na equação (3.8) é encontrada uma relação entre o tempo de vida da bateria $L$ e a carga varíável $i(t)$ no intervalo $[0, L]$ dada por

$$
\alpha=\sum_{k=1}^{n} 2 I_{k-1} A\left(L, t_{k}, t_{k-1}, \beta\right) .
$$

\section{Simulação dos Modelos Linear e de Rakhmatov- Vrudhula para uma Bateria Alcalina}

Nesta seção, é apresentado um estudo comparativo, a partir da realização de simulações, entre o modelo Linear e o modelo de Rakhmatov-Vrudhula, com o objetivo de demonstrar a diferença de consumo apresentado pela utilização do primeiro (i.e., Linear), que não considera os efeitos não-lineares no descarregamento da bateria, em relação ao segundo (i.e., Rakhmatov-Vrudhula), que considera tais efeitos. Neste contexto será utilizado um mesmo perfil de descarga, que contém as operações básicas $^{7}$ realizadas por um nó sensor.

\subsection{Definição do perfil de descarga}

Conforme mencionado anteriormente, será definido um perfil de descarga de uma bateria para ser utilizado nas simulações. Todos os parâmetros de simulação (Tabela 1) são obtidos a partir da especificação dos sensores da família Mica Motes [2]. A família Mica Motes é uma familia de nós sensores amplamente utilizada em pesquisas que envolve o conceito de Redes de Sensores Sem Fio (RSSF).

Para definir o tempo de transmissão e recepção que foram utilizados no perfil de descarga da bateria, foi considerada a taxa de transferência de dados dos sensores Mica Motes igual a 38,4 kbps e o tamanho do pacote de dados que teria que ser transmitido. Para este experimento foi definido um tamanho de pacote de 40 Bytes, o que significa que o tempo de transmissão e/ou recepção de um pacote de dados tem duração de aproximadamente $8 \mathrm{~ms}$.

Tabela 1: Parâmetros do Nó Sensor

\begin{tabular}{|c|c|}
\hline Subsistema & Especificação \\
\hline \hline Computacional & Modo Ativo $=8 \mathrm{~mA}$ \\
\hline Comunicação & Tx $=27 \mathrm{~mA}, \mathrm{Rx}=10 \mathrm{~mA}$ \\
\hline Fonte de Energia & 2 Baterias Alcalinas $\left(\mathrm{C}=2.459 .100 \mathrm{~mA}-\mathrm{ms}, V_{o c}=1.5 \mathrm{~V}, V_{c o}=0.8 \mathrm{~V}\right)$ \\
\hline
\end{tabular}

\footnotetext{
${ }^{7}$ Entende-se por operações básicas a transmissão e recepção de dados e também os momentos em que o nó sensor está em modo de espera (i.e., standby)
} 
Foi utilizado um perfil de descarga composto por um conjunto de 5 operações realizadas por um nó sensor, porém foram utilizadas apenas três modos (i.e., Transmissão (TX), Standby e Recepção (RX)) na seguinte sequência: Standby, TX, Standby, Rx e Standby. Os tempos, medidos em $m s$, de execução de cada operação e suas respectivas correntes, medida em $m A$, são listadas, respectivamente, nos vetores $P_{t}$ e $P_{i}$ listados a seguir:

$$
\begin{gathered}
P_{t}=\left[\begin{array}{lllll}
2 & 8 & 2 & 8 & 2
\end{array}\right]^{T}, \\
P_{i}=\left[\begin{array}{lllll}
8 & 27 & 8 & 10 & 8
\end{array}\right]^{T} .
\end{gathered}
$$

\subsection{Implementação dos modelos linear e de Rakhmatov-Vru- dhula}

O modelo analítico mais utilizado nas simulações do tempo de vida de uma bateria, é o modelo linear. Neste modelo a bateria é tratada como um recipiente linear de corrente. Assim, a equação

$$
C=C_{i}-I t_{d}
$$

permite calcular a capacidade restante $C$ de uma bateria, onde $C_{i}$ é capacidade no início da operação, $I$ é a corrente constante de descarga e $t_{d}$ é o tempo de duração da operação. A capacidade remanescente é calculada sempre que ocorrer mudança na taxa de descarga.

Já o modelo analítico de Rakhmatov-Vrudhula [10] foi originalmente desenvolvido para o cálculo do tempo de vida de uma bateria de íon(s) de lítio alimentada por carga constante ou variável. Na equação (3.10), considerando uma carga variável, é descrito o impacto do perfil de descarga no tempo de vida da bateria, onde $I_{k-1}$ é a corrente de descarga durante o período $k-1$. A função $A$ calcula o impacto do comportamento não-linear na descarga da bateria, onde $L$ é o tempo de vida da bateria, $t_{k}$ é o tempo de duração do período $k$, e $t_{k-1}$ é o tempo de duração para o período $k-1$. Mais detalhes sobre a função $A$ e sobre o algoritmo para calcular $L$ podem ser obtidos em [9].

Para estimar o tempo de vida de qualquer tipo de bateria, a partir do modelo de Rakhmatov-Vrudhula, somente dois parâmetros específicos são necessários, conforme é possível verificar na equação (3.10), o parâmetro $\alpha$ que está relacionado à capacidade da bateria e o parâmetro $\beta$ que está relacionado ao comportamento não-linear da bateria durante os períodos de carga e descarga. Neste trabalho, diferentemente do trabalho de Rakhmato-Vrudhula, utilizou-se uma bateria do tipo alcalina. Para tanto, foi necessário realizar a estimação dos parâmetros $\alpha$ e $\beta$ utilizando a mesma metodologia adotada por Rakhmatov-Vrudhula [9], que consiste em aplicar a técnica de Mínimos Quadrados a partir dos dados obtidos no site do fabricante da bateria [7]. Como resultado da estimação obteve-se os valores $\alpha=2.459 .100$ (i.e., capacidade da bateria) e $\beta=4.034$.

Ambos modelos foram implementados a partir da utilização da ferramenta matemática Matlab. A metodologia adotada foi simular a descarga da bateria utilizando o mesmo perfil nas implementações, comparando o resultado final da capacidade da bateria após um tempo de simulação de 60 segundos.

$\mathrm{Na}$ Figura 4 são apresentadas as capacidades residuais de uma bateria alcalina após a simulação do perfil de descarga $P_{i}$ durante 60 segundos, utilizando os modelos 
Linear e de Rakhmatov-Vrudhula. Verifica-se que o valor da capacidade residual, quando é utilizado o modelo de Rakhmatov-Vrudhula, é de $C r=2.451 .460,39$ $m A m s$, resultando em uma variação na capacidade de $C v=C_{0}-C_{f}=7.639,61$ $m A m s$ o que corresponde a uma redução de $0,32 \%$ na capacidade da bateria. Já a capacidade residual da bateria considerando a utilização do modelo Linear de descarga foi de $C r=1.517 .916 \mathrm{mAms}$ apresentando uma variação de $C v=941.184$ $m A m s$, o que corresponde a uma redução de $38,27 \%$ na capacidade da bateria.
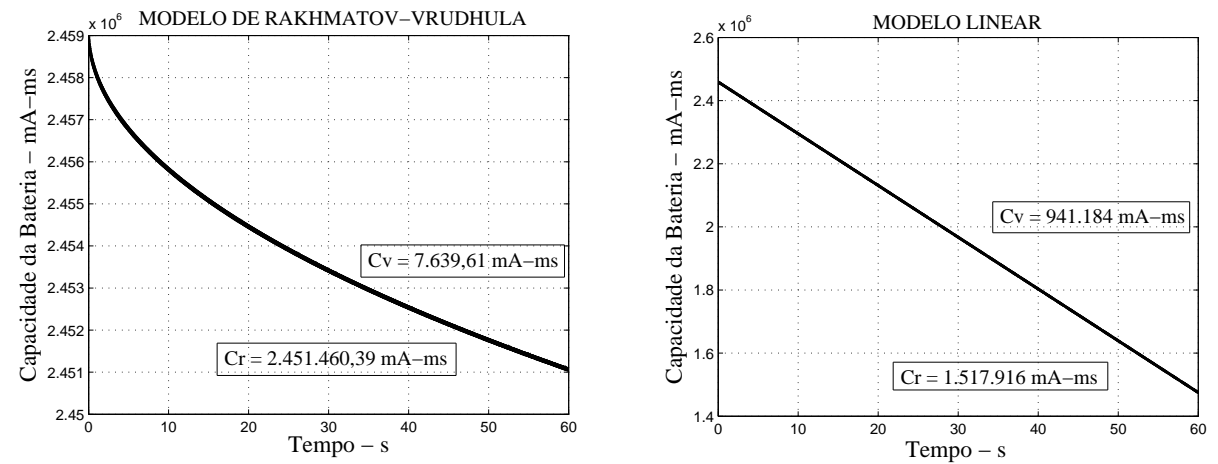

Figura 4: Capacidade remanescente da bateria utilizando o modelo de RakhmatovVrudhula e o Modelo Linear para tempo de simulação de 60 segundos.

A significativa variação na capacidade remanescente da bateria apresentado pelo modelo linear em comparação ao modelo de Rakhmatov-Vrudhula, ocorre devido ao modelo linear não levar em consideração os efeitos não-lineares que ocorrem durante a descarga da bateria (i.e., efeito de taxa de capacidade e efeito de recuperação) que foram detalhados na Seção 2 ..
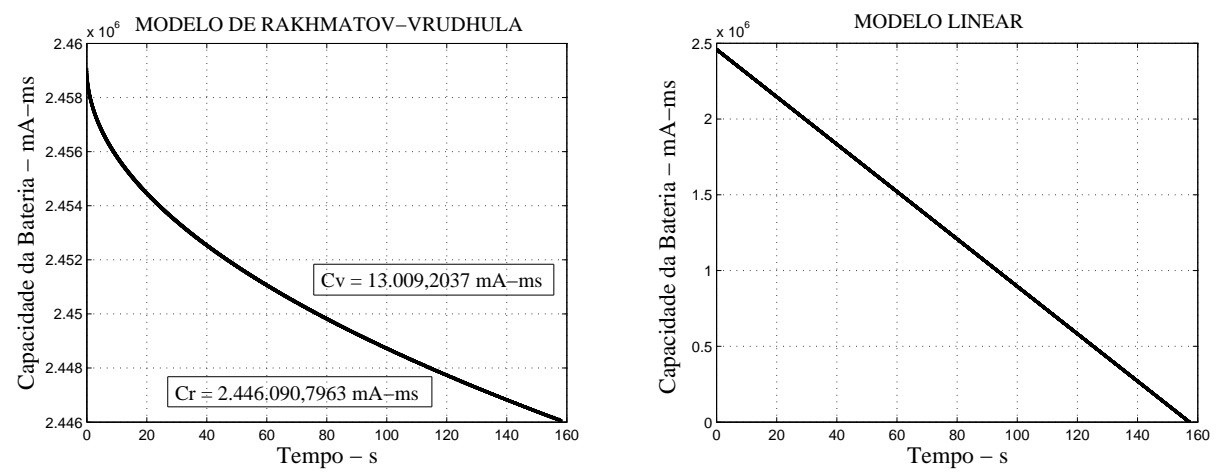

Figura 5: Capacidade remanescente da bateria utilizando o modelo de RakhmatovVrudhula e o Modelo Linear para tempo de simulação de 157 segundos.

A partir destes resultados pode-se verificar o quanto a adoção de um modelo não acurado, como é o caso do modelo linear, pode ocasionar interpretações equivocadas relacionadas com a capacidade remanescente de uma determinada bateria, uma vez que verificou-se que o modelo linear apresentou um consumo 123 vezes maior quando comparado ao modelo de Rakhmatov-Vrudhula para um mesmo perfil de 
descarga. Esta diferença fica mais evidenciada quando o tempo de simulação é estendido para 157 segundos e conforme pode-se verificar na Figura 5, o modelo linear descarrega completamente a bateria (i.e., capacidade igual a zero). Já realizando a mesma simulação, adotando o modelo de Rakhmatov-Vrudhula, a capacidade remanescente da bateria é de $C r=2.446 .090,7963 \mathrm{mAms}$, resultando em uma variação na capacidade de $C v=13.009,2037 m A m s$ o que indica uma utilização de apenas $0,52 \%$ da capacidade total da bateria.

\title{
5. Conclusões
}

Neste trabalho foram apresentadas a modelagem e simulação do consumo de energia de um nó sensor do tipo Mica Motes alimentado por uma bateria do tipo alcalina. Como estudo de caso foram comparados os resultados obtidos no cálculo da capacidade da bateria de um nó sensor através de dois modelos analíticos de bateria, o modelo linear, e um modelo mais acurado denominado modelo de RakhmatovVrudhula que, dentre outras características, considera os efeitos não-lineares durante o processo de descarga de uma bateria.

Os resultados obtidos têm mostrado que o modelo analítico de RakhmatovVrudhula apresenta resultados mais adequados para a estimação do tempo de vida de um nó sensor, pois consegue capturar o comportamento não-linear de uma bateria e a recuperação de sua capacidade durante períodos de alternância de correntes do nó sensor. Enquanto que o modelo linear, por ser bastante simples, não consegue capturar tais efeitos. Como trabalhos futuros, pretende-se estender o presente trabalho adicionando experimentos reais de descarga da bateria, comparando os resultados com os obtidos a partir das simulações realizadas neste trabalho.

\begin{abstract}
This paper presents a comparative analysis between two analytical models for the batteries discharge: the Linear model and the Rakhmatov-Vrudhula model. This analysis aims to verify which model is most appropriate for the lifetime estimation of sensor nodes powered by alkaline battery used in Wireless Sensor Networks (WSN). This way, simulations were performed considering the parameters of the alkaline battery and specifications of a sensor node of the family Mica Motes. From the simulation results analysis it was found that the Rakhmatov-Vrudhula model presented a smaller decrease in battery capacity when compared with Linear model, because it can capture the nonlinear effects that occur during the discharge operations.
\end{abstract}

\section{Referências}

[1] M. Chen, G.A. Rincón-Mora, Accurate electrical battery model capable of predicting runtime and I-V performance, IEEE Transactions on Energy Conversion, 21, No. 2 (2006)

[2] Crossbow, Crossbow technology inc. data sheet, http://www.xbow.com, 2010.

[3] M.R. Jongerden, B.R. Haverkort, Which battery model to use? in "Proceedings of the 24th UK Performance Engineering Workshop", pp. 76-88, London, 2008. 
[4] M.R. Jongerden, B.R. Haverkort, "Battery Modeling", Technical Report in Faculty Electrical Engineering, Mathematics and Computer Science, 2008.

[5] K. Lahiri, A. Raghunathan, S. Dey, D. Panigrahi. "Battery-Driven System Design: A New Frontier in Low Power Design", Dept. of ECE, UC San Diego, La Jolla, Research Labs, NEC USA, Princeton, NJ.

[6] T.L. Martin, "Balancing Batteries, Power, and Performance: System issues in cpu speed-setting for mobile computing", Ph.D. dissertation, Carnegie Mellon University, 1999.

[7] Panasonic, Panasonic industrial alkaline batteries data sheet, http://www.panasonic.com/industrial/batteries-oem/specialty-products/alkaline.aspx, 2010.

[8] D. Panigrahi, C. Chiasserini, S. Dey, R. Rao, A. Raghunathan, K. Lahiri, "Battery Life Estimation of Mobile Embedded Systems", NEC USA Inc. and the California Micro Program.

[9] D. Rakhmatov, S. Vrudhula, An analytical high-Level battery model for use in management of portable electronic systems, in "Proceedings of ICCAD", pp. $1-6,2001$.

[10] D. Rakhmatov, S. Vrudhula, Energy management for battery-powered embedded systems, ACM Transactions on Embedded Computing Systems, 2, No. 3 (2003), 277-324.

[11] P. Sausen, "Gerenciamento Integrado de Energia e Controle de Topologia em Redes de Sensores Sem Fio", Tese de Doutorado, Universidade Federal de Campina Grande, Campus de Campina Grande, PB, 2008. 